\title{
Revealing the secrets of composite helmets of ancient Japanese tradition
}

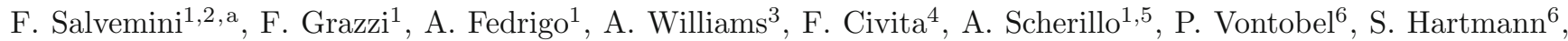 \\ E. Lehmann ${ }^{6}$, and M. Zoppi ${ }^{1}$ \\ 1 Consiglio Nazionale delle Ricerche, Istituto dei Sistemi Complessi, 50019 Sesto Fiorentino (FI), Italy \\ 2 Università di Firenze, Dipartimento di Scienze della Terra, Italy \\ 3 Wallace Collection, W1U 3BN London, UK \\ ${ }^{4}$ Museo Stibbert, 50100 Firenze, Italy \\ 5 Science and Technology Facility Council, ISIS Neutron Source, OX11 0QX Didcot, UK \\ ${ }^{6}$ Paul Scherrer Institut, SINQ Neutron Source, 5232 Villigen, Switzerland
}

Received: 28 February 2013 / Revised: 28 May 2013

Published online: 12 August 2013 - (c) Società Italiana di Fisica / Springer-Verlag 2013

\begin{abstract}
We present novel results from a non-invasive examination of two kabuto (helmets), made in Japan in the 17th century. Neutron-imaging experiments (radiography and tomography), carried out at the ICON and NEUTRA beamlines, operating at the neutron source SINQ $(\mathrm{CH})$, have allowed to determine the inner metal structure and manufacturing techniques of these beautiful examples of past technology, revealing some otherwise invisible details.
\end{abstract}

\section{Introduction}

Japanese swords and armours have always been very attractive to the Western culture because of their distinctive styles, which are considerably different from the corresponding objects familiar to Western culture. However, the selfevident differences in style correspond to the technological differences that material scientists can help to understand. To this aim, scientific research applied to this class of samples would help in elucidating the forging techniques, which where used by ancient Japanese craftsmen in the manufacture of sword blades and armours. Much literature exists about Japanese swords, but far less is known about the technology of armours. So, an international team of scientists and curators decided to work together, to investigate the construction of one of the most critical components of the Japanese armour, namely the helmet (kabuto).

The kabuto of the traditional samurai armour is a kind of helmet, typically made of steel components, assembled in ways that are peculiar to the particular manufacturing school. These objects are quite rare and, when found in museums, are usually in an excellent state of conservation, being considered masterpieces representative of the Japanese culture. For this reason, any detailed study of these artefacts must rely on non-invasive techniques and it was decided that thermal and cold neutron techniques should be employed for this investigation.

Among the various components of the samurai's armour, the helmet assumes, for obvious reasons, considerable importance. Here, the technological skill of the craftsman might reach the best results in joining lightness and effectiveness to defend the most important organ of the samurai's body: the head. In addition, being the most visible part of the warrior from a distance, the helmet assumed also the role of the distinctive sign of a leader in battle. Thus, not only effectiveness, but also elegance and visibility became necessary qualities for the samurai's helmet.

The kabuto became an important part of the traditional Japanese armour during the feudal period, and was initially worn only by high-ranking warriors [1,2]. During the Momoyama Period (1568-1603), armourers began to build fantastic shapes on top of the earlier simple helmets, in order to improve visibility and enhance the leader's presence on the battlefield. These kabuto were composed of a steel bowl, surmounted by a structure of papier mâché and lacquer, or sometimes entirely of iron. These shapes mimicked forms taken from Japanese culture and mythology, including fish, shells, dragons, etc. [2]. The kabuto can be classified into three main categories: those made from a

\footnotetext{
a e-mail: floriana.salvemini@fi.isc.cnr.it
} 

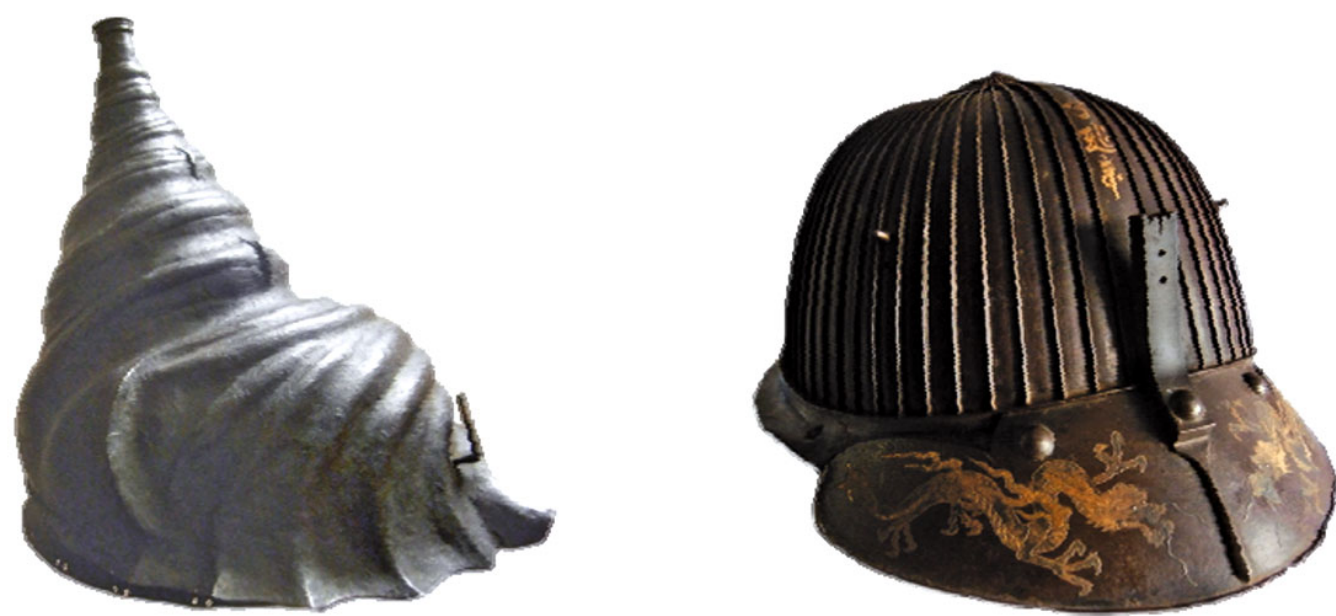

Fig. 1. The picture on the left shows the unconventional shape of the horagai bachi helmet (shell shaped). On the right, the suji-bachi helmet is shown: this is a multiple-plate type of hachi (Japanese helmet bowl) with raised ribs on every plate and connection rivets hidden into the internal structure.

single sheet of metal, folded and welded; those assembled from a small number of sheets; and those assembled from a large number (varying between 8 and 128) of lamellar plates arranged in a circular fashion around the crown on top of the head [2]. From the mid 16th century, when a Portuguese ship first landed on Tanegashima Island, Japan came into contact with the European culture, tradition, art, and fighting techniques. As a result, the armament production was strongly influenced and armour construction and design, including helmets, were modified in an attempt to resist firearms $[2]$.

For the analysis presented in this work, we have selected two examples of ancient helmets. In fact, the two chosen kabuto are antithetical in their complexity: while the first is characterized by a unusually complex shape, the second is made of a large set of relatively simple components, assembled in a very complex structure. Each one was dated by the museum experts using stylistic analysis and archive studies.

The helmet shown on the left side of fig. 1 is a horagai bachi, a peculiar example of a helmet of the 1st type (as previously defined). This is classified as a tetsu bari shiki kawari-bachi (wonderfully shaped steel helmet) and was made, in the form of a shell, by Ryōei - Ohara Katsunari from Hiroshima province at the end of the 17th century. This helmet was made available by the Stibbert Museum in Florence.

The kabuto shown on the right-hand side of fig. 1 is, according to our definition, an example of the third type. This is a signed Saotome bachi made in the 1st half of the 17th century by Saotome Ienari. He was the third craftsman of the Saotome dynasty, so the helmet is one of the oldest surviving of this type. The sample is made of 64 lamellar plates, finished with a visor decorated with two facing gold dragons. It is a typical suji kabuto, or "flange helmet bowl", presenting ribs at the edge of each plate. The various components are joined by rivets invisible because the surface is lacquered on the inside and patinated on the outside. This helmet was kindly provided by a British private collector and was formerly part of the H. Russel Robinson Collection ${ }^{1}$.

\section{Scientific investigations}

Neutrons, thanks to their high penetration power in dense matter [3], represent an almost unique method for the non-invasive characterization of the microstructure of massive metal objects [4]. Neutron diffraction techniques have been recently applied to the study of metal artefacts of archaeological and historical origin, and have given detailed information on bulk properties (e.g., phase composition, texture, residual strain distribution), which has allowed us to obtain information on the manufacturing techniques from crystallographic details [5]. On a much larger length scale, neutron-imaging techniques [6] can add useful information about the bulk conservation status and the inner structure of the artefact [7-12]. Neutron imaging can be used for non-destructive evaluation of such inner properties starting with single radiographies up to 3D tomographic reconstructions, obtained by multiple image acquisitions.

\footnotetext{
${ }^{1}$ H. Russell Robinson was an expert in Roman armours and curator at the Royal Armouries of Britain. After his death, the collection was spread and acquired by other private collectors.
} 


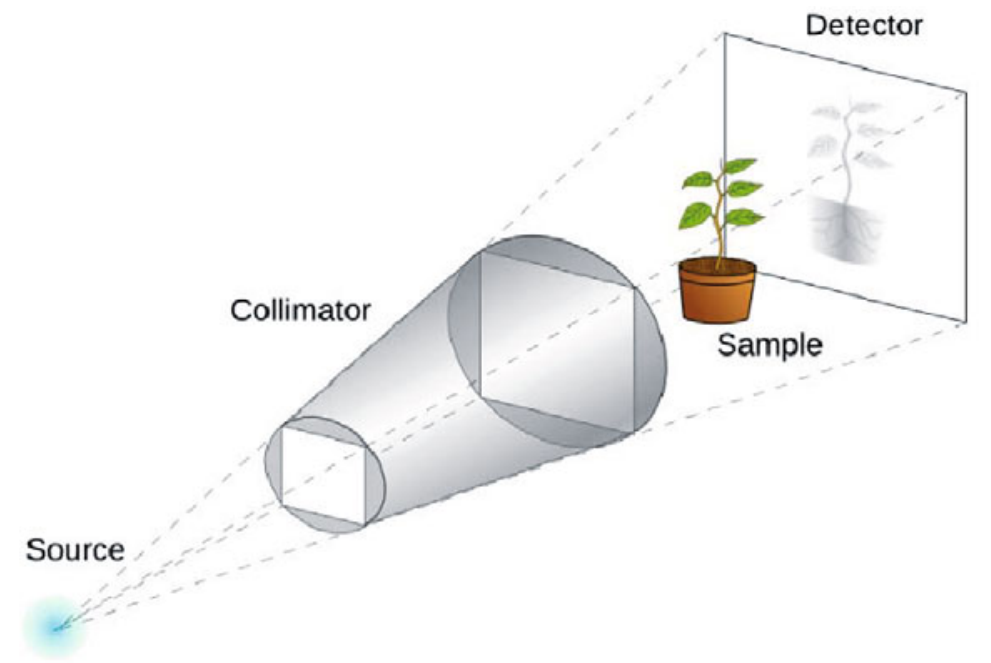

Fig. 2. A schematic view of a basic neutron-imaging experimental set-up. The radiation is generated by a neutron source and is properly collimated. The achievable image resolution depends much on the collimator geometry: the $L / D$ ratio, where $L$ is the length of the collimator and $D$ the diameter of its inlet aperture, is widely used as a measure for the beam divergence. The primary beam transmitted through the sample is recorded by a flat position-sensitive detector. The detector records a two-dimensional image which is the projection of the object on the detector plane [15] (http://www.psi.ch/niag/neutron-imaging-setup).

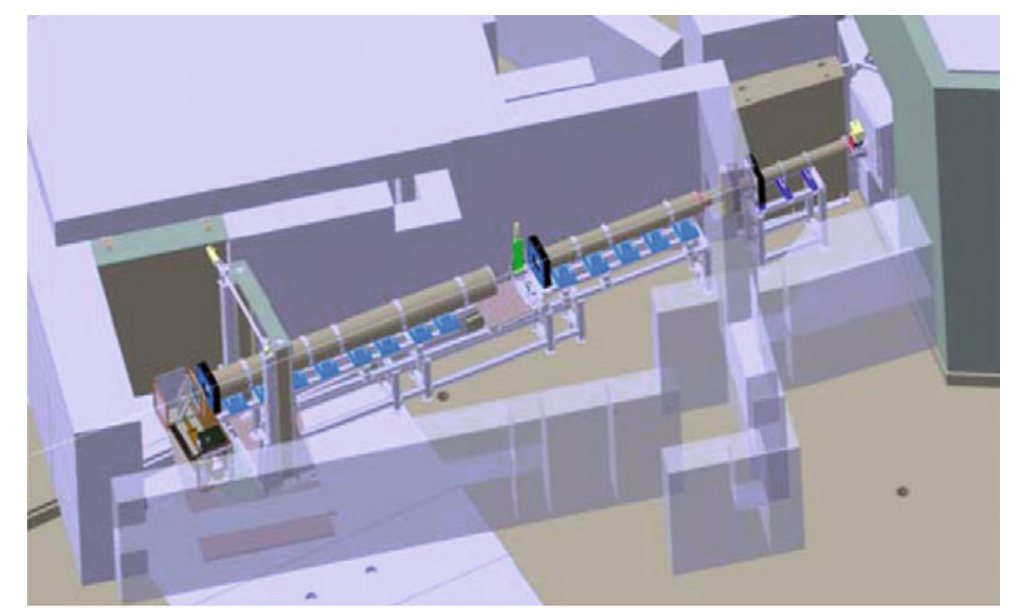

Fig. 3. The ICON beamline at SINQ (http://www.psi.ch/sinq/icon).

The neutron radiography method is based on the application of the basic law of radiation attenuation [13,14]. When the investigated object is exposed to the beam, neutrons interact differently with the nuclei of the atoms composing the sample, according to the total cross-section of the constituent elements and the flight path across the analysed object [15]. The emerging neutrons, detected by a two-dimensional position-sensitive detector (fig. 2), generate an optical image that contains information about material conformation and the structure inside the sample [16]. In our experiment, the neutron radiography has been performed at the ICON facility [17], using the neutron beam originating from the cold liquid- $\mathrm{D}_{2}$ moderator (sketch given in fig. 3), at the spallation neutron source SINQ (CH) (http://www.psi.ch/sinq).

The peculiar cold neutron wavelength of the ICON instrument allowed us to enhance the contrast between the different phases and the different thickness of the material, thanks to the large cross-sections for some elements and the consequent high sensitivity in thin layers.

In fig. 3, the experimental beamline ICON at SINQ $(\mathrm{CH})$ is shown, where the horagai-bachi neutron radiographies were taken.

Tomographic techniques provide sliced images of an object from the transmission data obtained by combining cross-section measurements taken by exposing the sample in many different orientations. The method can be readily applied to bulk metal samples using thermal and cold neutrons, which are detected using a proper scintillator plate. In order to reconstruct the three-dimensional volume map of the sample, a series of 2D parallel projections are measured rotating the object around its vertical axis over a range of (at least) $180^{\circ}$. 


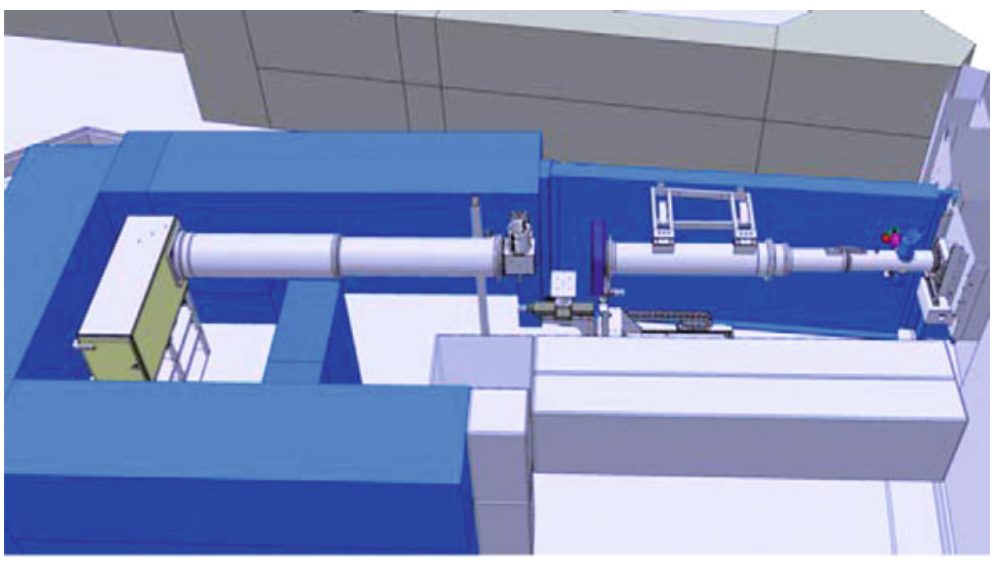

Fig. 4. The NEUTRA bamline at SINQ (http://www.psi.ch/sinq/neutra).

Table 1. The different instrumental set-up employed for measurements of the two Japanese helmets.

\begin{tabular}{|l|l|l|}
\hline Sample & Horagai Bachi helmet & Suji-Bachi helmet \\
\hline Instrument & ICON & NEUTRA \\
\hline Method & Neutron radiography & Neutron tomography \\
\hline Experimental positions & 3 (distance $L$ from the beam port exit $=12.10 \mathrm{~m})$ & $\begin{array}{l}3 \text { (distance } L \text { from the beam port } \\
\text { exit }=10.547 \mathrm{~m})\end{array}$ \\
\hline Aperture sizes $D$ & $20 \mathrm{~mm}$ & $20 \mathrm{~mm}$ \\
\hline$L / D$ & 605 & 550 \\
\hline Step angle & - & $0.576^{\circ}$ \\
\hline Rotating angle & - & $360^{\circ}$ \\
\hline Number of projections & 13 & 625 \\
\hline Exposure time for radiography & $12 \mathrm{~s}$ & $20 \mathrm{~s}$ \\
\hline Camera & Andor DV434 - BV, 16 bit & Andor iKon-L DW936N, 16 bit \\
\hline CCD size & $1024 \times 1024$ pixel & $2048 \times 2048$ pixel \\
\hline Scintillator & ${ }^{6}$ LiF $/$ ZnS $(1: 2) 100 \mu \mathrm{m}$ & 6 LiF $/$ ZnS $(1: 2) 200 \mu \mathrm{m}$ \\
\hline Field of view & $323.4 \times 323.4 \mathrm{~mm}$ & $309 \times 309 \mathrm{~mm}$ \\
\hline Lens optic system & $50 \mathrm{~mm} \mathrm{Nikkor} \mathrm{lens}$ & $50 \mathrm{~mm}$ Nikkor lens \\
\hline Object-detector distance & $150 \mathrm{~mm}$ & $148 \mathrm{~mm}$ \\
\hline Resulting spatial resolution & $310 \mu \mathrm{m}$ & $150 \mu \mathrm{m}$ \\
\hline
\end{tabular}

After corrections of the raw images (i.e. neutron exposure, flat-field correction, bright spots filtering, rotation axis positioning), the tomographic slices are calculated using the inverse radon transform (filtered back-projection) [18]. Finally, all the slices are collected in an image stack representing the 3D volume data of the neutron attenuation properties of the sample. At the end of the process, the different parts of the object with different neutron attenuation characteristics can be visualized using a proper 3D rendering software [19,20]. The neutron tomography study has been performed using the NEUTRA facility [21], the thermal neutron imaging station at SINQ.

In order to cover the whole volume of the helmet, within the field of view of the instrument (NEUTRA), the sample was placed diagonally over an aluminium cylindrical frame and gently fixed using aluminium strips. The very low cross-section of aluminium makes this metal almost transparent to neutrons, thus avoiding the need of a complex data analysis to remove interferences between the two components during the data reduction. The acquired neutron tomography data were processed through the software package Octopus, developed at the Ghent University [22].

In fig. 4, the experimental beamline NEUTRA (also at SINQ) is shown, where the suji-bachi neutron tomography was recorded.

In table 1 the different experimental configurations and instrument set-up for the two measured samples are summarized. 
$\mathbf{a}$

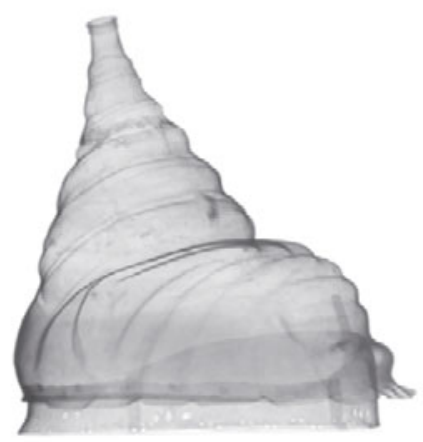

c

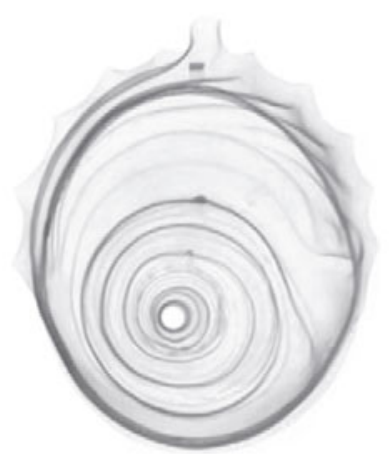

b

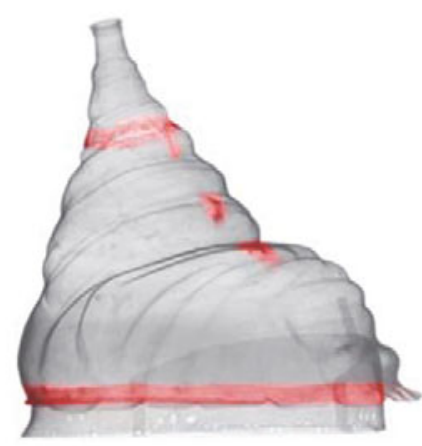

d

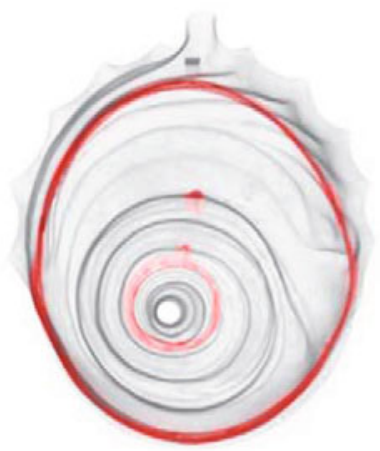

Fig. 5. Selected neutron radiographies show the helmet structure. In (a) and (c) the transversal and bottom views, respectively; in (b) and (d), the brazing positions linking the three different metal portions are marked in red (see text).

We point out that dealing with precious museum objects implies taking extra precautionary measures preventing possible damage to the sample or somehow impairing its availability for a much too long time (e.g., because of a long-living residual radiation activity) [5]. To this aim, the first kabuto (the shell-shaped horagai bachi) was initially characterized trough a preliminary Neutron Activation Analysis test measurement. This analysis was used to evaluate the concentration of trace and major elements (and their isotopes) in the sample with the aim of testing its activation rate and decay time as a function of the exposure time [23]. In order to limit the activation level induced by the presence of $\mathrm{Cu}$ and to avoid a decay time longer than a few hours, this helmet was investigated for a limited neutron exposure time and only 13 neutron radiographies were taken from different positions on the sample. No such problems emerged in the analysis of the second kabuto (the bell-shaped suji-bachi) and therefore, in this case, a full tomographic study was possible.

\section{Results}

The following images show some of the neutron radiographies and neutron tomography reconstructions of the two helmets. The non-invasive analysis allows the identification of some peculiarities of the manufacturing methods used by the Japanese craftsmen. In particular, details of some specific structural elements shed some light on the assembly techniques of the objects.

\section{Horagai bachi helmet (shell shaped)}

The set of neutron radiographies allowed us to discover that this helmet is built of three different metal sectors and these were joined (by brazing) in such a way that it was impossible to see the seams due to the surface finish patina. The seams are shown in fig. 5 enhanced by the red lines. The first circular brazing, close to the top, is connecting the upper almost conical part of the helmet to the lower portion. The second brazing is linear and closes the seam of the large single-plate component bent to form the central part of the helmet. Finally, a third circular brazing was 


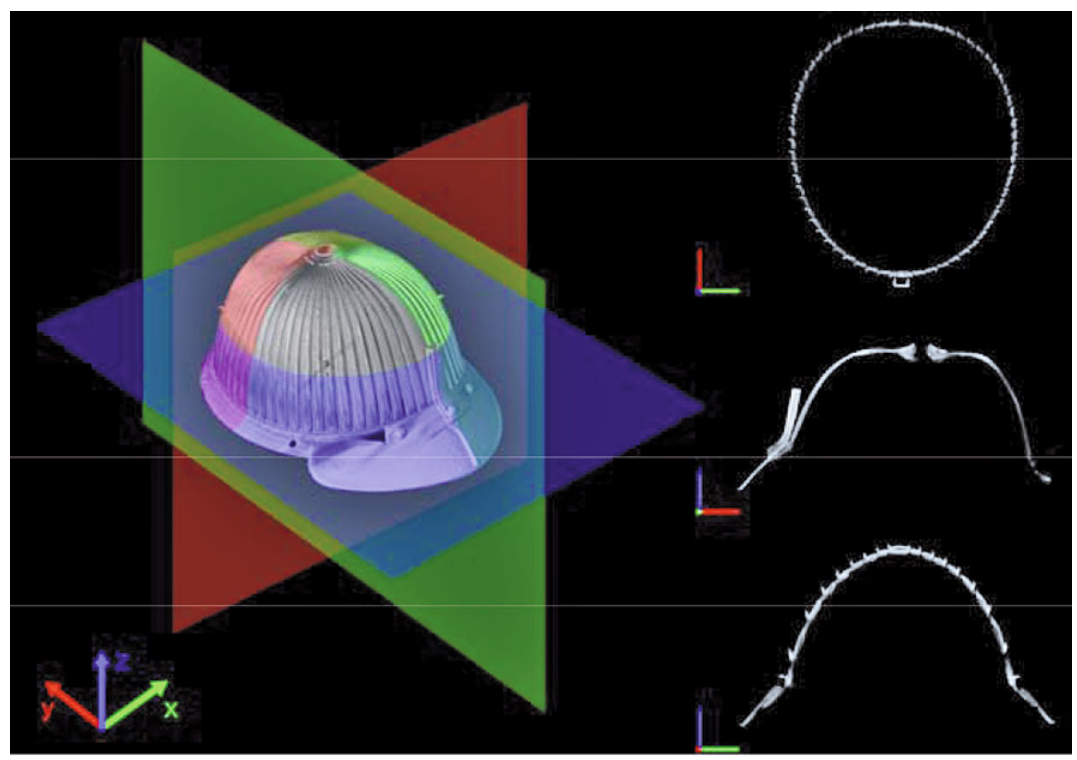

Fig. 6. On the left, perpendicular sections trough the helmet (blue, green, and red planes) and their orientation ( $x-, y_{-}$, and $z$-axis) within the virtual 3D space. From top to bottom on the right side, axial, transversal, and normal views clearly show how the tomographic images were used to gain information on the kabuto inner structure.

used to connect the body of the helmet with the circular plate on the bottom. It should be noted that the brazing was absolutely invisible, and could only have been detected by removing the original patina, which covers the kabuto surface both internally and externally. A careful analysis of the gamma emission spectrum originating from the brazing material allowed us to identify it as pure copper.

\section{Suji-bachi helmet (bell shaped)}

Concerning the Saotome bachi helmet, the tomographic reconstruction of the kabuto allowed us to acquire a wealth of information about the inner structure and assembly methods of this masterpiece. In the left half of fig. 6 , we report the kabuto image and the reference system of the tomographic planes. In the right part, the 3D tomographic image is cut along the selected planes and the resulting images are shown. From top to bottom, we observe: 1) the horizontal (axial) section obtained by slicing along the blue plane; 2) the longitudinal section obtained by slicing along the green plane; 3) the transversal section obtained by slicing along the red plane.

The helmet axial section, reported magnified in fig. 7(a), is further magnified in fig. 7(b). From these images (and from others, even further magnified) the actual arrangement of the lamellar components in the kabuto was made visible. The construction starts from the central plate, in the back of the helmet. The S-shaped vertical plates overlap one other, leaving an empty space in the centre of the superimposed area. This arrangement was invented to absorb the energy of a blow and was probably evolved from a simpler structure such as that one shown in the upper drawing of fig. 7 (c), where flat plates are fixed to one another by rivets (boshi). According to the current literature [1], the vertical curved plates of the helmet (tate hagi-no-ita) are interconnected by orthogonal rivets as well. These are supposed to be inserted through the centre of a portion of each lower plate and connecting to the centre of the curved portion of the upper plate (see the intermediate drawing in fig. $7(\mathrm{c})$ ). Instead, in our case, the image reconstruction shows a different layout characterized by diagonal boshi, as reported in the bottom scheme of fig. 7(c).

This type of arrangement shows well in the orthogonal projections of the tomographic reconstruction of fig. 8. Such a structure allows the helmet to be more resistant to blows. In fact, if the helmet is struck, every plate involved tends to transfer the impact to the next ones. This permits the differently curved parts to contribute to the absorption of the impact energy. Moreover, the external ribs located in the side end of the plates are facing towards the back of the helmet. Thanks to this arrangement, most of the impact energy is transferred to these ribs in case of a frontal or diagonal hit. The diagonal orientation of the rivets helps in keeping the structure stable under the blows offsetting their tendency to deform. It is amazing how a simple modification in the alignment of the boshi improves all the intrinsic advantages of the curved structure by adding stability hence strength to the entire structure.

We also observed that the central plate located in the back of the kabuto is composed by a single metal sheet whose edges are completely covered by the two adjacent sectors (see upper part of fig. 7(a)). On the contrary, the frontal area was manufactured by superimposing a properly curved narrow metal sheet over a large 3-folded one that completes the round shape of the helmet, thus fixing and securing both sides of the hachi (helmet bowl) structure (see fig. 7(b)). 
a

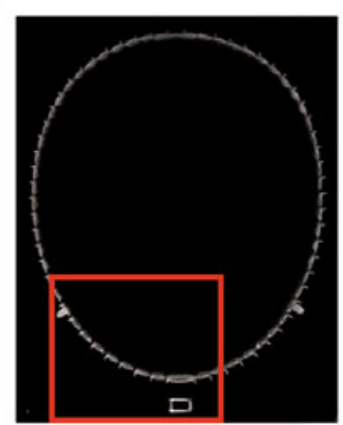

c

Helmet structure reported in the literature

Detected helmet structure in this case b
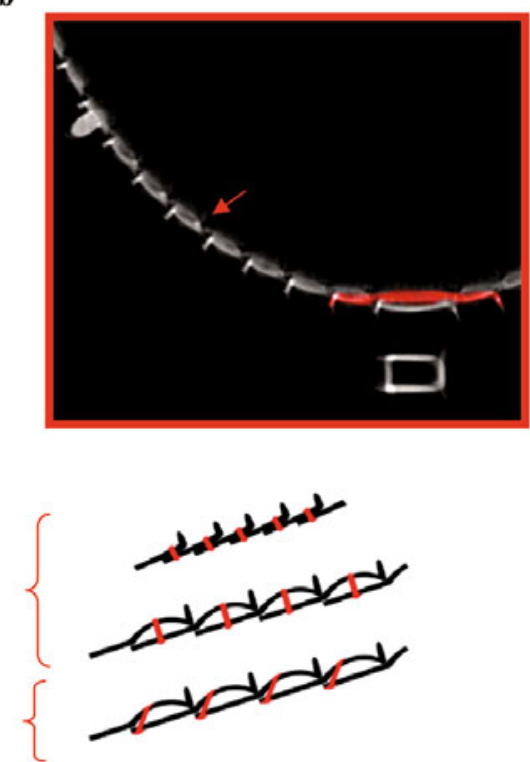

Fig. 7. The image (a) is the axial cross-section taken half way up through the helmet; the red rectangle marks the magnified region reported in (b). The magnification clearly shows the arrangement of the overlapped S-shaped plates. The red arrow points out the position of a rivet, linking the extreme end of the lower plate with the central part of the upper one. The frontal area is manufactured superimposing a properly curved narrow metal sheet over a large 3-folded one, here coloured in red. As shown in picture (c), the detected structure is of a new type with respect to the two kinds so far reported in the literature.

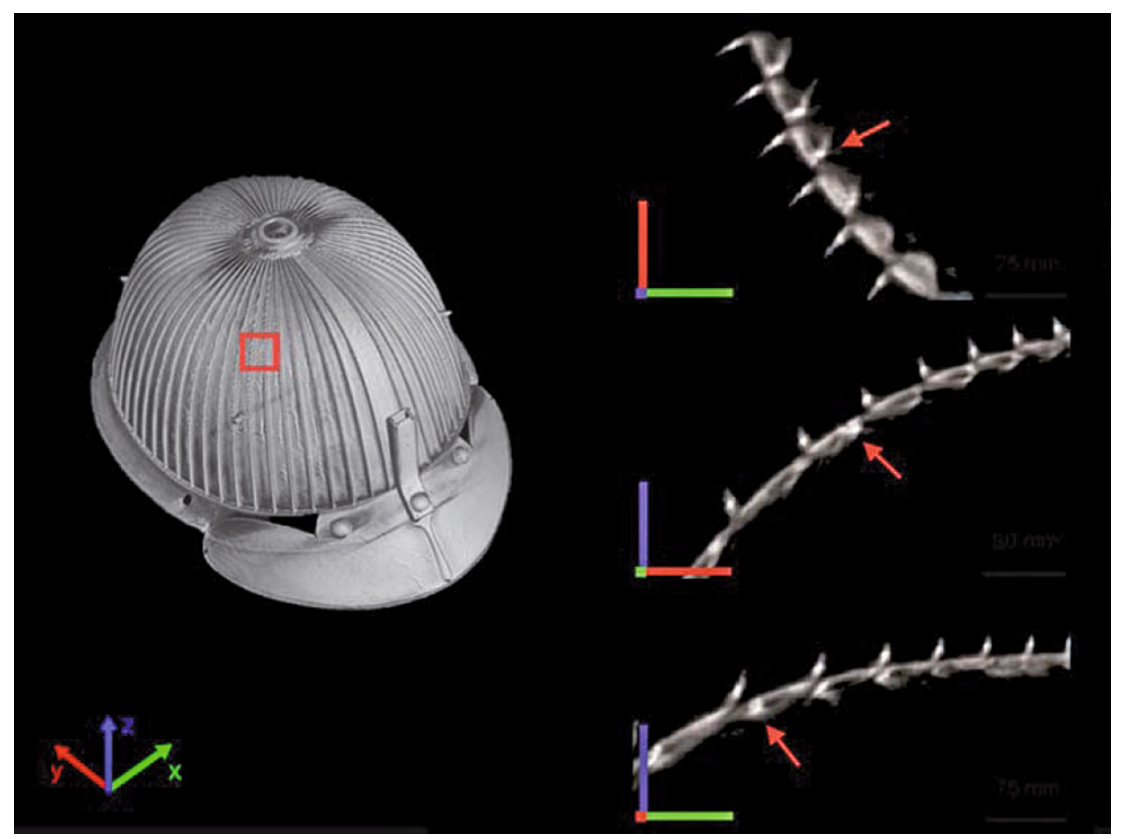

Fig. 8. In the 3D reconstructed image, the red square marks the position of a hidden rivet. On the right, from top to bottom, the red arrows point its precise location in the axial, transversal and normal orthogonal views, respectively.

The rivets are arranged in six concentric rings running at different heights all around the helmet circumference joining adjacent plates, holding together and stabilizing the two-layer structure.

As can be observed in fig. 9 (an enlarged view of the transversal section already shown in fig. 6) all the plates radiate vertically from a central opening at the top of the bowl, named tehen. This portion of the kabuto is marked with the upper red rectangle, while the lower one depicts a schematic view. In the same figure, we note that the visor (tsuke mabizashi) and the front crest holder (haraidate-dai) were fixed together and anchored to the helmet body through a large and prominent rivet called hachi tsuke-no-byo. 


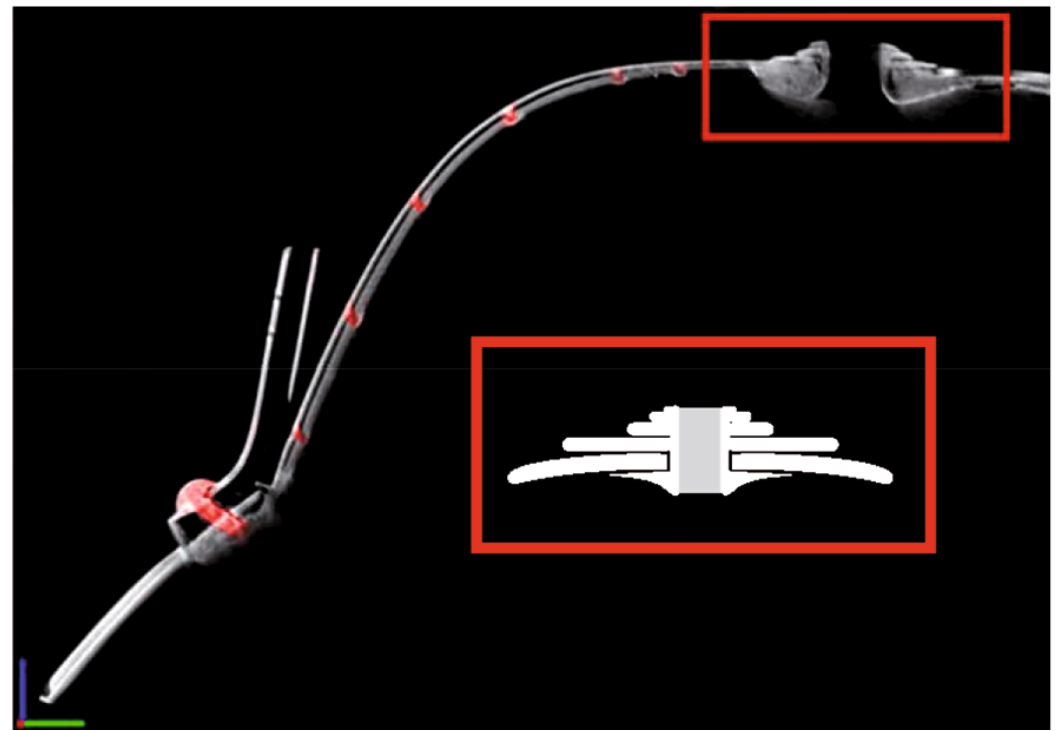

Fig. 9. Normal section taken through the middle of the helmet: red spots map the rivets position along the two central superimposed lamellar plates. An anchor rivet, also in red, joins the visor and the front crest holder to the hachi. The upper red frame points out the position of the tehen with its idealized structure depicted in the lower red rectangle.

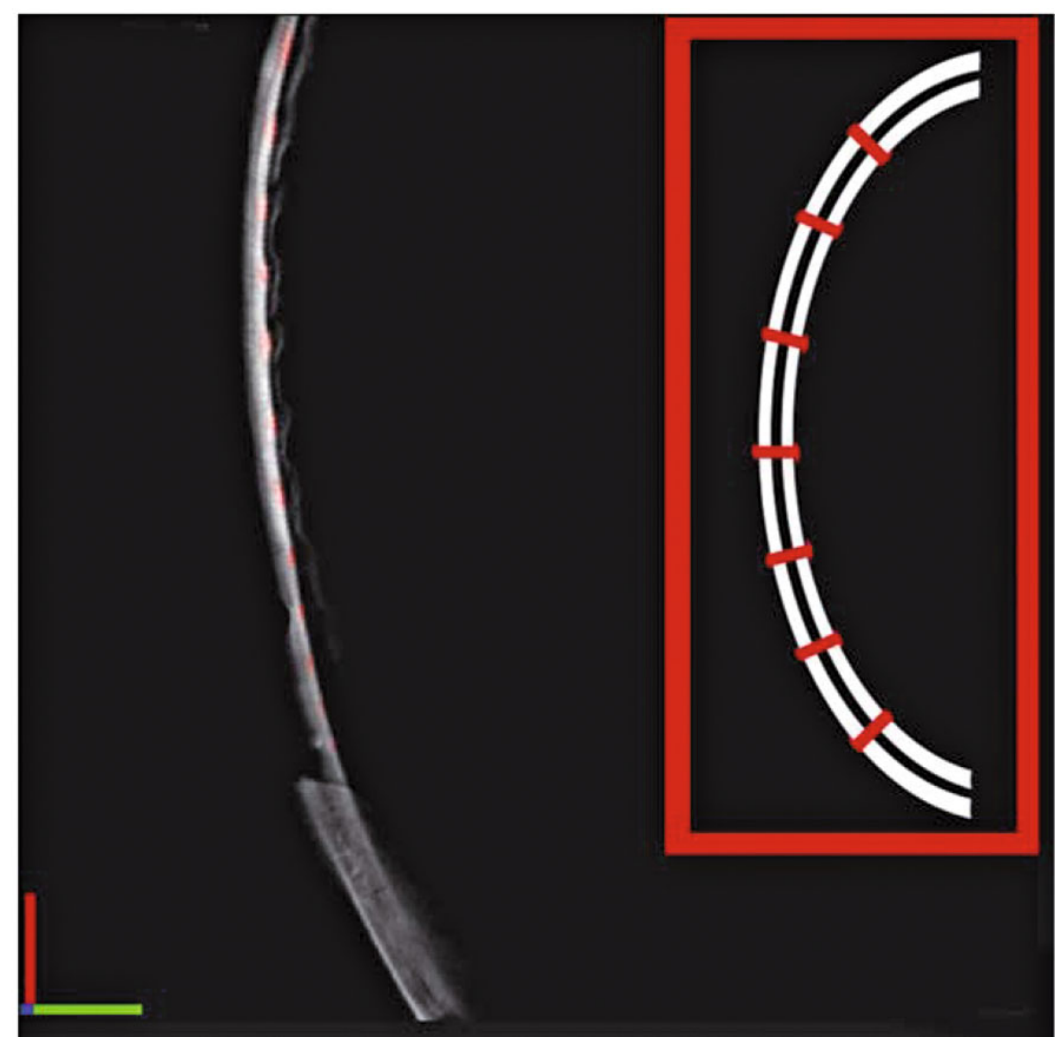

Fig. 10. The axial cross-section at the bottom of the helmet shows the arrangements of the koshimaki and the hachi. The light red spots mark the rivets positions, as shown by the scheme in the red frame on the right.

The round plate at the bottom of the helmet is named koshimaki and is attached to the upper part (hachi) with a single line of rivets. This is depicted in fig. 10. On the left side, the tomographic image of this section is shown, while on the right, in the red frame, a scheme of the two curved portions of the koshimaki and of the hachi shows the connecting rivets in red. 
a

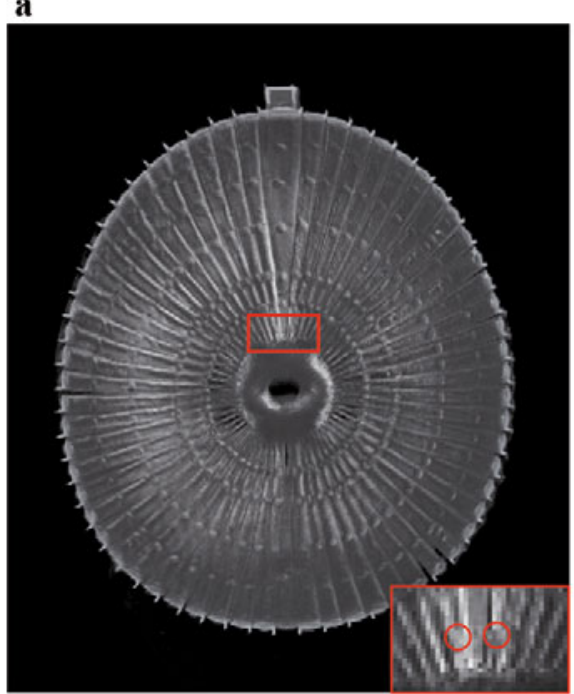

b

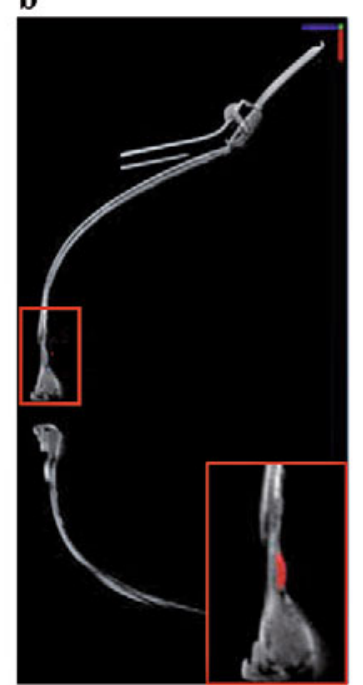

Fig. 11. The axial 3D cross section (a) allows to define, looking from the bottom, the byo position in the inner-upper portion of the central plate. On the right (b), the red rectangle points out the rivets location in the transversal section. At the right-side bottom of each image, a magnification of the region of interest shows byo marked in red.

Another important and unexpected feature revealed by the tomographic reconstruction of this kabuto is the presence of two byo, whose position is evidenced in fig. 11 by the two red circles (picture on the left) and by the rectangles (picture on the right). These two rivets have no structural function but were typically placed by the maker as his own signature. The number and position of these byo, characteristic of the Saotome school, confirmed the helmet attribution that was previously based only on the available documents and the stylistic analysis.

\section{Conclusions}

This work is the result of a collaborative investigation carried out on two Japanese helmets belonging to different structural categories from the 17th century. The present analysis could not have been carried out using traditional (invasive) analytical methods, which cannot be applied to such ancient and rare artefacts where a non-invasive, nondestructive experimental approach is mandatory.

Neutron-imaging experiments were used to identify the peculiar characteristics of the construction methods used for two different types of kabuto. In particular, in the horagai bachi helmet more than one metal plate component was revealed and the invisible brazing lines were identified.

Concerning the suji bachi helmet, a truly complex assembly method was elucidated:

- the presence, the location, and the direction of insertion of hidden rivets were determined;

- the hidden lamellar plate shape was established and a novel arrangement discovered;

- the inner structure, resulting from the peculiar manufacturing process, was analysed and the assembly of tehen, tsuke mabizashi, and hachi was identified.

The present results show that the proposed investigation technique, using neutron imaging, allowed us revealing unexpected features with respect to the current literature reports. This opens new interesting perspectives and further work will be needed to gain a more thorough understanding of the ancient art of Japanese armour making. In particular, more samples need to be analysed to extend our knowledge to the wide variety of kabuto types, as well as of other armour components.

The success of the experiment was largely obtained through a close collaboration between scientists and museum curators. We strongly believe that this is the key to further progress in this kind of studies.

The authors gratefully thank Jonathan Barrett for illuminating discussions. This research project has been supported by the European Commission under the 7th Framework Programme through the "Research Infrastructures" action of the "Capacities" Programme, NMI3-II Grant number 283883. 


\section{References}

1. Sakakibara Kozan, The manufacture of armour and helmets in 16th century Japan: (chūkokatchü seisakuben), revised and edited by Russel H. Robinson (The Holland Press, London, 1963).

2. C. Sinclaire Samurai: The Weapons and Spirit of the Japanese Warrior (The Lyons Press, Guilford, 2004).

3. V.F. Sears, Neutron News 3, 26 (1992).

4. S. Siano et al., Appl. Phys. A 74, S1139 (2002).

5. A. Fedrigo et al., J. Anal. At. Spectrom. 286, 908 (2013).

6. N. Kardjilov, I. Manke, A. Hilger, M. Strobl, J. Banhart, Mater. Today 14, 248 (2011).

7. L. Cartechini et al., J. Phys. Condens. Matter 20, 1 (2008).

8. E.H. Lehmann, P. Vontobel, E. Deschler-Erb, M. Soares, Nucl. Instrum. Methods A 542, 68 (2005).

9. F. Grazzi, L. Bartoli, F. Civita, M. Zoppi, Anal. Bioanal Chem. 395, 1961 (2009).

10. E.H. Lehmann, S. Hartmann, M.O. Speidel, Archaeometry 52, 416 (2010).

11. F. Grazzi et al., J. Anal. At. Spectrom 26, 1030 (2011).

12. F. Salvemini et al., J. Anal. At. Spectrom. 27, 1494 (2012).

13. J.H. Lambert, Photometria sive de mensura et gradibus luminis, colorum et umbrae ("Photometry or on the measure and gradations of light, colors, and shade") (Augsburg "Augusta Vindelicorum", Germany: Eberhardt Klett, 1760).

14. A. Beer, Ann. Phys. Chem. 86, 78 (1852).

15. D.C. Mannes, Non-Destructive Testing of Wood by means of Neutron Imaging in comparison with similar methods, A dissertation submitted to Eth Zurich for the Degree of Doctor of Sciences, Dipl. Forstwirt, Albert-Ludwigs-Universität Freiburg I. Br. (D).

16. J.C. Domanus, Commission of the European Communities, Euratom, Neutron Radiography Working Group, in Practical Neutron Radiography (Springer, 1992).

17. A.P. Kaestner et al., Nucl. Instrum. Methods Phys. Res. A 659, 387 (2011).

18. F. Natterer, The Mathematics of Computerized Tomography, in Classics in Applied Mathematics, Vol. 32 (Society for Industrial and Applied Mathematics, 1986) ISBN 0898714931.

19. E.H. Lehmann, S. Hartmann, M.O. Speidel, Archaeometry 52, 416 (2010).

20. L. Giller, U. Filges, G. Kühne, M. Wohlmuther, L. Zanini, Nucl. Instrum. Methods Phys. Res. A 586, 59 (2008).

21. E. Lehmann, P. Vontobel, L. Wiezel, Nondestr. Test Eval. 16, 191 (2001).

22. M. Dierick, B. Masschaele, L. Van Hoorebeke, Meas. Sci. Technol. 15, 1366 (2004).

23. Z.B. Alfassi, Activation Analysis, Vols. I and II (CRC Press: Boca Raton, FL, 1990). 